Check for updates

Cite this: RSC Adv., 2017, 7, 32155

Received 30th April 2017

Accepted 11th June 2017

DOI: $10.1039 / c 7 r a 04843 b$

rsc.li/rsc-advances

\title{
Designing waterproof breathable material with moisture unidirectional transport characteristics based on a TPU/TBAC tree-like and TPU nanofiber double-layer membrane fabricated by electrospinning
}

\author{
Jingge Ju, ${ }^{\text {ab }}$ Zhijie Shi, ${ }^{\text {ab }}$ Nanping Deng, ${ }^{\text {ab }}$ Yueyao Liang, ${ }^{\text {ab }}$ Weimin Kang*ab \\ and Bowen Cheng (D) *ab
}

\begin{abstract}
In this study, a thermoplastic polyurethane (TPU) tree-like nanofiber membrane was fabricated via one-step electrospinning by adding a small amount of tetrabutylammonium chloride (TBAC). On the basis of the "push and pull" effect, double-layer membranes composed of pure TPU nanofiber membranes (hydrophobic) and TPU/TBAC tree-like nanofiber membranes (hydrophilic) were prepared by the direct electrospinning compounding method. The double-layer membranes were used as waterproof breathable materials with moisture unidirectional transport properties and good shielding properties. The water resistance, mechanical, waterproof, moisture permeability, air permeability, air filtration and moisture unidirectional transport performances of the double-layer membranes were tested. The results showed that the double-layer TPU membranes displayed good performances compared with the existing products on the market; they provide a new approach for the development of waterproof breathable materials.
\end{abstract}

\section{Introduction}

At present, people's requirements for clothing are not simply aesthetic; the function and comfort of clothing are equally important. Waterproof breathable clothing is a representative of this kind of clothing; it is known as the second skin of human beings, and breathable fabric. A waterproof breathable material combines waterproof properties and moisture permeability in a functional fabric, ${ }^{1}$ and it plays an important role in special protective clothing, outdoor recreational clothing and other materials. $^{2-6}$ Traditional waterproof breathable materials include high-density fabric, coated fabric and laminated fabric. However, their methods of preparation need to be improved in many respects, and it is difficult for them to meet the higher level requirements of the new types of waterproof breathable materials.

Compared with traditional waterproof breathable materials, electrospinning nanofiber membranes have small apertures, high specific surface areas and abundant internal porous channels, which can isolate any damage effectively and offer good air and moisture permeability at the same time. The

${ }^{a}$ State Key Laboratory of Separation Membranes and Membrane Processes, Tianjin Polytechnic University, Tianjin 300387, China. E-mail: kweimin@126.com; bowen15@tjpu.edu.cn

${ }^{b}$ School of Textiles, Tianjin Polytechnic University, Tianjin 300387, China electrospinning nanofiber membranes also offer good wearability due to their resilience and low coefficients of friction. ${ }^{7}$ Moreover, the electrospinning technology is a simple, low-cost and high-tech method which can prepare nanofibers directly and continuously and so it has drawn attention from many researchers. ${ }^{8-12}$ Some high-performance multi-level structured nanofibers with high specific surface areas and high porosities have been prepared by one-step electrospinning ${ }^{13-18}$ and have been widely used in the information, energy ${ }^{19,20}$ and environmental ${ }^{21,22}$ industries as well as in other fields. ${ }^{23}$ Kang et al. ${ }^{24}$ examined the feasibility of electrospinning polyurethane onto substrate fabrics to prepare waterproof breathable fabrics. Lee et al. ${ }^{25,26}$ investigated the changes in the mechanical properties and thermal and water transfer properties of a mass-produced nanofiber web after laundering, to evaluate the possibility of using nanofibers for outdoor wear. Yoon and $\mathrm{Lee}^{27}$ developed waterproof breathable materials for diverse consumer applications, and they used electrospinning to fabricate layered fabric systems with varying composite structures. From the functional perspective, waterproof breathable fabric can block external water and harmful particles. From the comfort perspective, people need to transmit water vapour out and maintain a comfortable small climate zone when exercising or working under special circumstances. Research into waterproof and permeability properties has been increasingly developed, but there have been few studies on the moisture unidirectional 
transport of functional materials based on these two fundamental properties. The goal is a kind of functional material which can discharge sweat and water vapour generated by the human body to the outside while preventing external water and harmful particles entering into the clothing and contacting the human body. ${ }^{28}$

Dong et $a .^{29}$ prepared a composite double-layer fiber membrane composed of thick hydrophilic polyacrylonitrile (PAN) and thin hydrophobic polystyrene (PS) through electrospinning. Then the double-layer membrane was coated with polydopamine (PDA), and a porous structure was obtained for the PS fiber membrane. This could enhance the hydrophobicity difference for moisture transport. The unidirectional transport of moisture from the PS layer to the PAN layer was achieved, but there was no evaluation of moisture permeability or waterproofing properties. Therefore, it would be of profound significance to prepare a kind of high-performance waterproof breathable material with functions of both protection and moisture unidirectional transport.

In order to achieve waterproof, moisture permeable and moisture unidirectional transport functions, a composite material composed of at least two heterogeneous layers with different performances is needed. In this work, a double-layer membrane composed of a hydrophilic TPU/TBAC tree-like nanofiber membrane and a hydrophobic pure TPU nanofiber membrane was designed by direct combination using electrospinning. The water resistance, mechanical, waterproof, moisture permeability, air permeability, air filtration and moisture unidirectional transport performances of the double-layer membranes were characterized to obtain the optimal membrane thickness. Moreover, the properties were compared with those of a commercial PU waterproof breathable membrane.

\section{Experimental}

\section{Materials}

Thermoplastic polyurethane (TPU) was purchased from the Lubrizol Corporation (Wickliffe, Ohio, USA). N,N-dimethylformamide (DMF) and tetrabutylammonium chloride (TBAC) were purchased from Tianjin Kermel Chemical Reagent Co., Ltd. (China). All materials were purchased commercially and were used without further purification. Distilled water was used in this work.

\section{Method}

Preparation of TPU/TBAC tree-like nanofiber membrane. The spinning solutions were prepared by dissolving TPU (7 $\mathrm{wt} \%$ ) and TBAC ( $1 \mathrm{wt} \%)$ in DMF with vigorous stirring over $10 \mathrm{~h}$. TBAC was added in order to obtain nanofibers with tree-like structures. $^{30}$ In order to reduce the solution viscosity and increase the solubility of TPU, a small amount of LiCl was introduced into the solution, ${ }^{31,32}$ and then the mixed spinning solution was transferred into a syringe $(10 \mathrm{~mL})$. A voltage of 45 $\mathrm{kV}$ was applied in the experiment using a high voltage power supply (DW-P503-4ACF1, Tianjin, China). The outer diameter of the needle was $0.8 \mathrm{~mm}$, and a syringe pump (WZ-50C2) was

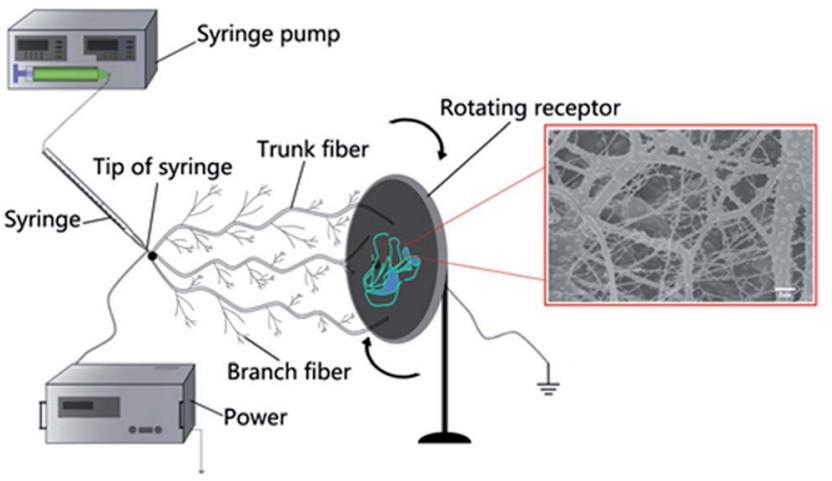

Fig. 1 A schematic diagram of electrospinning.

used to control the feed rate of the solutions at $0.5 \mathrm{~mL} \mathrm{~h}^{-1}$. The TPU/TBAC tree-like nanofiber membranes (denoted TPU/TBACTLNMs) were received on the aluminium foil-covered rotating receptor at the selected distance of $10 \mathrm{~cm}$ between the tip of the syringe and the collector (Fig. 1). The formation mechanism of the tree-like nanofibers was attributed to the introduction of TBAC. TBAC, as a kind of organic branched salt, easily dissolved in the spinning solution and significantly increased its electrical conductivity and decreased its viscosity. ${ }^{33}$ When the excess charge density at the bending segments was above a certain threshold value, the electric forces overcame the surface tension, leading to the splitting of the jet and causing the formation of tree-like structures. In addition, the TBAC reduced the forces between the PU molecules because of its space steric structure which was beneficial for the slipping of the jets. ${ }^{34}$

Preparation of pure TPU nanofiber membrane. The spinning solution was prepared by dissolving TPU (7 wt\%) in DMF with vigorous stirring over $10 \mathrm{~h}$. Then, the spinning solution was transferred into the syringe and the electrospinning process was carried out with appropriate parameters (voltage of $35 \mathrm{kV}$, feed rate of $1.0 \mathrm{~mL} \mathrm{~h}^{-1}$ and collected distance of $10 \mathrm{~cm}$ ). Finally, the TPU nanofiber membranes (denoted TPU-NMs) were obtained.

Preparation of double-layer membrane. The double-layer membrane was prepared by firstly electrospinning TPU-NMs on aluminium foil and then by directly electrospinning TPU/TBACTLNMs. The TPU/TBAC-TLNMs formed the outer membrane close to the environment and the TPU-NMs formed the inner membrane close to the skin, and these membranes were bonded together tightly. The TPU/TBAC-TLNMs with their special treelike morphology should bring good moisture conductivity to the double-layer membrane. Combined with the good hydrophobicity of TPU, the "push-pull effect" should be produced such that the moisture is conducted more effectively from the inner layer to the outer layer (unidirectional moisture transport). ${ }^{35} \mathrm{~A}$ schematic diagram of this process is shown in Fig. 2.

Characterization. The surface morphologies of the nanofiber membranes were characterized by field-emission scanning electron microscopy (FE-SEM, Hitachi S-7400, Hitachi, Japan). The diameters of the fibers and pore sizes of the membranes were measured by an image analyzer (IPPwin32, Soft Imaging System) on a sample of 100 fibers. The pore size and 


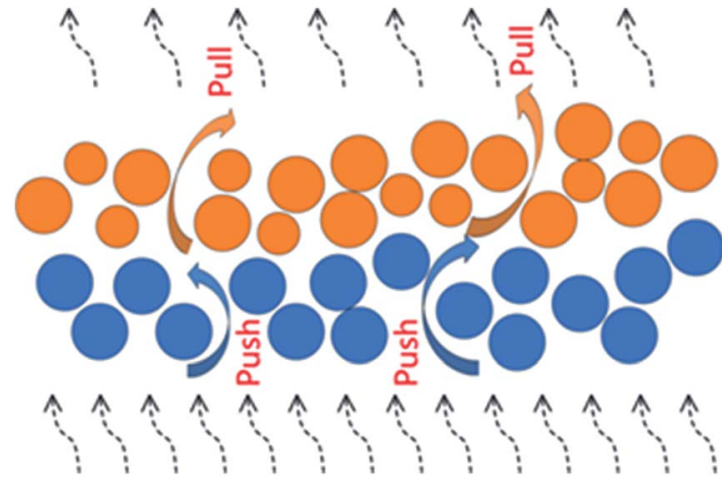

Fig. 2 A schematic diagram of the "push-pull effect".

distribution of the double-layer membrane was analyzed by a pore size meter (PSM-165, Topas, GmbH, Germany). The water contact angles (WCA) of each sample were analyzed by a JYSP180 contact angle tester (Jinshengxin Detection Instrument Co., Ltd., Beijing, China) equipped with a high-resolution TV camera. The mechanical properties of the membranes were measured with a monofilament tensile testing machine (YG 005E, Wenzhou Fangyuan Instrument Co., Ltd., China), at a speed of $10 \mathrm{~mm} \mathrm{~min}^{-1}$ at room temperature. The moisture transport behavior of the membranes was tested using a moisture management tester (MMT, SDL ATLAS). The friction coefficient of the nanofibrous membranes was tested using a Tribometer (CSM Instruments). The washing resistance performance of the double-layer membrane was measured with the following conditions: water temperature of $40{ }^{\circ} \mathrm{C}$, phosphorus-free ECE standard detergent, drying at room temperature, flat state. The water vapour transmission of the membranes was investigated by using a YG501D testing chamber. The hydrostatic pressure of the membranes was measured using a M232 spray rating tester (SDL ATLAS). The breathability of the membranes was measured using an automatic air permeability instrument (YG461H). The filtering quality was tested using an automatic filtering tester (8130, TSI Corp., America).

\section{Results and discussion}

\section{The surface morphology and pore size investigations}

As shown in Fig. 3a, the different sides of the double-layer membrane presented varying morphologies. The TPU/TBACTLNMs side displayed tree-like fibers (Fig. 3a1), while the fibers in the TPU-NMs were flat without branches (Fig. 3a2). The pore sizes of TPU/TBAC-TLNMs ranged from $0.2 \mu \mathrm{m}$ to $2.5 \mu \mathrm{m}$ (mainly focused at around $0.3 \mu \mathrm{m}$ ), and the pore sizes of TPUNMs ranged from 0.4 to $4.5 \mu \mathrm{m}$ (mainly focused at around 0.5 $\mu \mathrm{m})$ as shown in Fig. $3 \mathrm{~b}$. To verify the reliability of the data, the pore size distribution of the double-layer membrane was measured using the Topas aperture instrument which revealed a range from $0.4-1.8 \mu \mathrm{m}$ (mainly focused at $0.4-0.6 \mu \mathrm{m}$ ), as shown in Fig. 3c. This result was consistent with the previous data. In theory, the double-layer membrane could prevent the
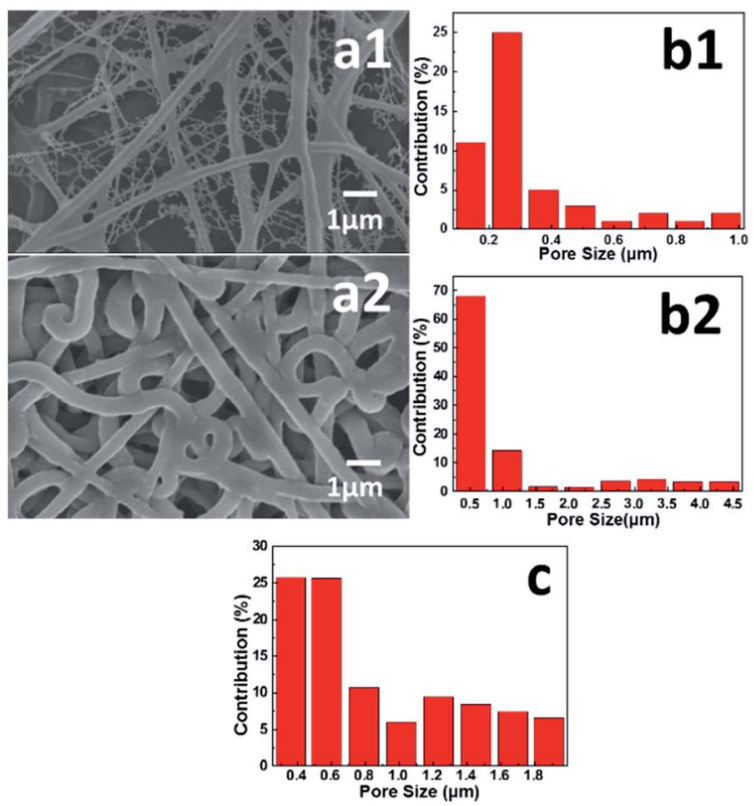

Fig. 3 (a) SEM images and (b) pore size distributions from the doublelayer membrane: (1) TPU/TBAC-TLNMs (outside, close to the environment) and (2) TPU-NMs (inside, close to the skin); (c) pore size distributions of the double-layer membrane.

penetration of water droplets (100-3000 $\mu \mathrm{m})$ and allow the penetration of water vapour $(0.0004 \mu \mathrm{m})$ according to the pore permeability mechanism.

\section{The contact angles analysis}

To verify the hydrophobicity on each side of the membrane, the contact angles were tested. According to Fig. 4a and b, the single TPU-NMs layer (the inner layer which contacted the skin) was hydrophobic, and the single TPU/TBAC-TLNMs layer (the outer layer which was exposed to the atmosphere) was hydrophilic.
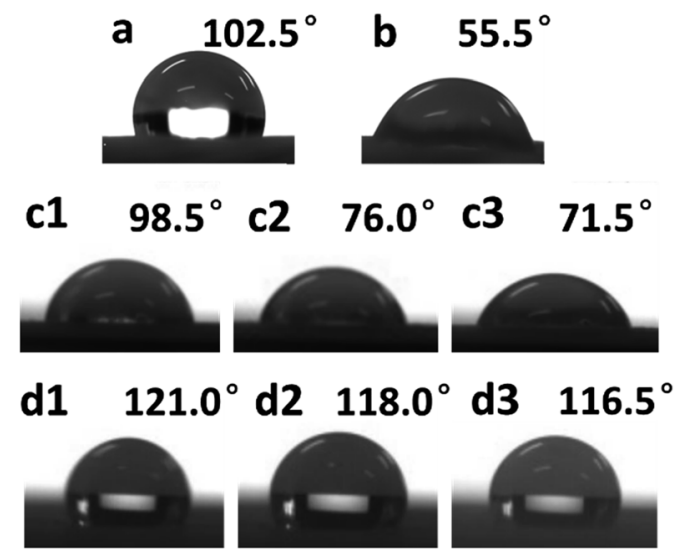

Fig. 4 The contact angles of (a) TPU-NMs; (b) TPU/TBAC-TLNMs; and ( $c$ and d) the double-layer membrane: (c) the TPU/TBAC-TLNMs side (close to the environment) and (d) the TPU-NMs side (close to the skin). Measurements were taken at (1) $0 \mathrm{~s}$, (2) $5 \mathrm{~s}$ and (3) $10 \mathrm{~s}$ after water droplets were applied. 


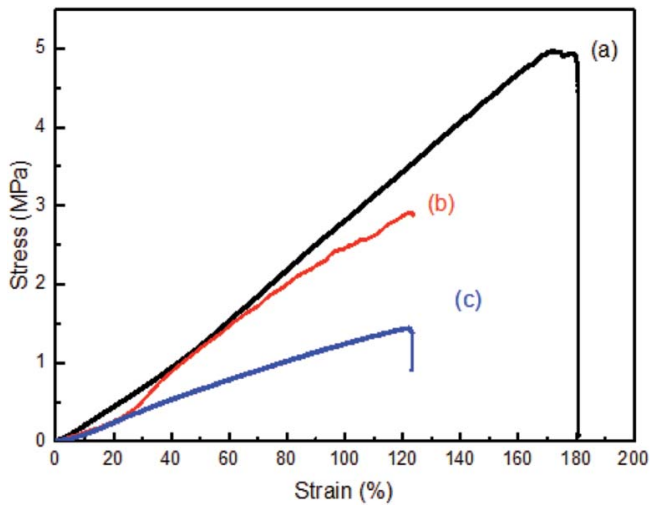

Fig. 5 The stress-strain curves of (a) TPU-NMs, (b) the double-layer membrane and (c) TPU/TBAC-TLNMs.

The TPU-NMs layer was hydrophobic because of the properties of TPU. The introduction of TBAC brought with it a strong hydroxyl group which could effectively improve hydrophilicity. ${ }^{36}$ Moreover, the formation of the tree-like structure had a capillary effect, and the presence of the tree-like structure with its greater surface area increased the surface energy to make it more hydrophilic. As shown in Fig. $4 \mathrm{c}$ and d, the contact angles of the double-layer membrane were obtained at $0 \mathrm{~s}, 5 \mathrm{~s}$ and $10 \mathrm{~s}$ after applying water drops. The two sides of the double-layer membrane exhibited different results. The obtained contact angles after $10 \mathrm{~s}$ were $116.5^{\circ}$ on the TPU-NMs side and $71.5^{\circ}$ on the TPU/TBAC-TLNMs side, which confirmed that the different surfaces of the double-layer membrane showed varying contact angles.

\section{The mechanical properties test}

The stress-strain curves of TPU-NMs, TPU/TBAC-TLNMs and the double-layer membrane are shown in Fig. 5. It is clear that the TPU-NMs had the highest tensile strength (4.46 MPa) compared with the other membranes; the fine branches in the other membranes would not offer so much mechanical strength because of their higher elongation. The TBAC/TPU had decreased tensile strength $(1.44 \mathrm{MPa})$ because the addition of TBAC increased the solution conductivity and led to the accumulation of fluffy fibers. Meanwhile, the double-layer membrane was prepared by combining TPU-NMs and TPU/ TBAC-TLNMs, and it had medial tensile strength (2.9 MPa) which effectively solved the problem of the insufficient strength of TPU/TBAC-TLNMs.

\section{The studies of unidirectional moisture transporting properties}

The Moisture Management Tester (MMT) images of different TPU-NMs thicknesses $(0.08 \mathrm{~mm}, 0.04 \mathrm{~mm}$ and $0.02 \mathrm{~mm})$ are shown in Fig. 6a-c, and the MMT image of TPU/TBAC-TLNMs is shown in Fig. 6d. The MMT images reflect the membrane moisture transport capacity; they include curves of water absorption against time and the intuitive pictures of effective wetting. The black sections are the regions in which there was no wetting, and the blue regions show the water absorbing capacity of the membranes. When the membrane thickness was $0.04 \mathrm{~mm}$, water could not be transported from the top surface to the bottom surface which demonstrated that the membrane had a good waterproofing function and moisture transport capacity. However, when the membrane thickness was 0.08 $\mathrm{mm}$, the membrane could not be made wet on either side because of over thickness, and the $0.02 \mathrm{~mm}$ membrane was so thin that it was difficult to achieve a waterproof effect. The experimental results confirmed that the moisture transmission ability was weakened with the increase of membrane thickness, and that the $0.04 \mathrm{~mm}$ thickness was the best choice.

The water transporting data from the different TPU-NMs and TPU/TBAC-TLNMs are listed in Table 1 . The wettability and moisture transport performance of both sides of each membrane were evaluated by six parameters: wetting time (WT), maximum absorption rate (MAR), maximum wetting radius (MWR), water spread speed (SS), overall wet transport capacity (OWTC) and overall moisture management capacity (OMMC). The OWTC and OMMC evaluate the overall moisture unidirectional transport ability of the membrane, and larger numerical values mean stronger moisture unidirectional transport ability. It can be seen that the TPU-NMs and TPU/TBAC-TLNMs had no moisture unidirectional transport ability.

In order to verify the unidirectional moisture transport property of the double-layer membrane, the MMT experiment was performed on the outer and the inner sides of the membrane, respectively. To achieve the best moisture transport capability of the double-layer membrane, the average membrane thickness used in the experiment was $0.12 \mathrm{~mm}$ (the TPU-NMs layer was $0.04 \mathrm{~mm}$, and the TPU/TBAC-TLNMs layer was $0.08 \mathrm{~mm}$ ), and the results are shown in Fig. 7 and Table 2 . As shown in Fig. 7a, the water was dripped to the TPU/TBACTLNMs side. It was found that the TPU/TBAC-TLNMs side could be made wet but that the water could not penetrate into the TPU-NMs side. Although the TPU/TBAC-TLNMs layer was hydrophilic, it had a microcellular structure which could prevent the water from getting through. Moreover, the water was obstructed by the TPU-NMs side which was hydrophobic, and the waterproof function was achieved. As shown in Fig. 7b, water could be transported to the outside and evaporate in the atmosphere when it was dripped to the TPU-NMs side. Water could not wet the double-layer membrane and was pulled by the hydrophilic TPU/TBAC-TLNMs, which proved the "push-pull effect" principle. Therefore, the double-layer membrane realized the function of unidirectional moisture transportation. The total unidirectional overall wet transport capacity of the doublelayer membrane was $34.9324 \%$.

\section{The friction coefficient survey}

The friction coefficient is one of the important indexes used to measure the comfort of clothing. The friction resistance produced by the clothing can cause mild damage to the skin and hinder wearability, so the frictional resistance needs to be controlled within an appropriate range. The friction coefficient is about 0.4 in common clothing made from cotton. The 

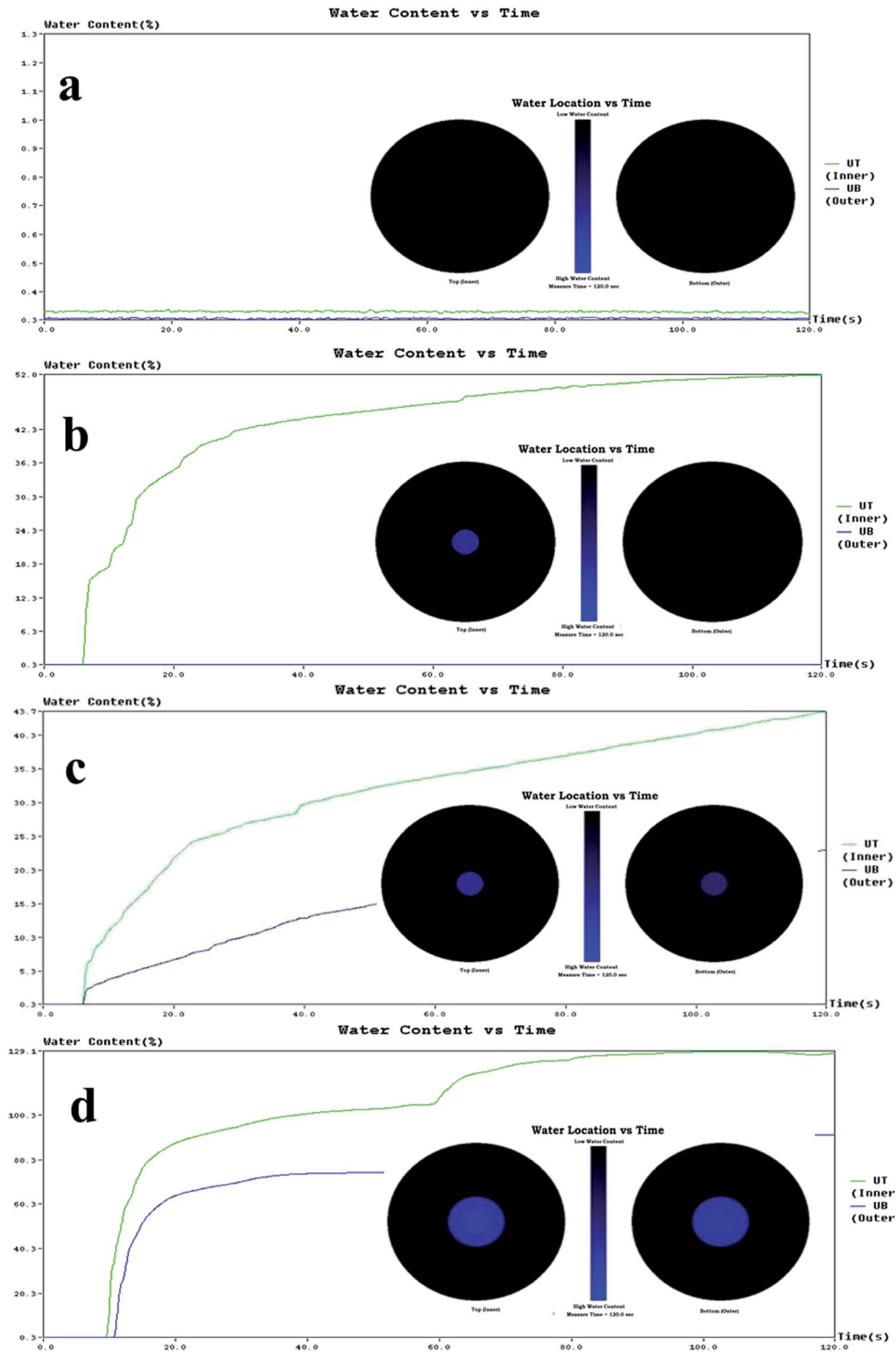

Fig. 6 MMT images of (a-c) TPU-NMs with different thicknesses: (a) $0.08 \mathrm{~mm}$, (b) $0.04 \mathrm{~mm}$ and (c) $0.02 \mathrm{~mm}$; (d) TPU/TBAC-TLNMs (0.08 mm).

comparison of friction coefficients among the prepared membranes and cotton is shown in Fig. 8. It can be seen that the prepared double-layer membranes have a similar friction coefficient to that of cotton, while the friction coefficient of TPU/TBAC-TLNMs is 0.74 which is larger than those of the other membranes. This may be because the TPU/TBAC-TLNMs 
Table 1 MMT test data for single-layer membranes

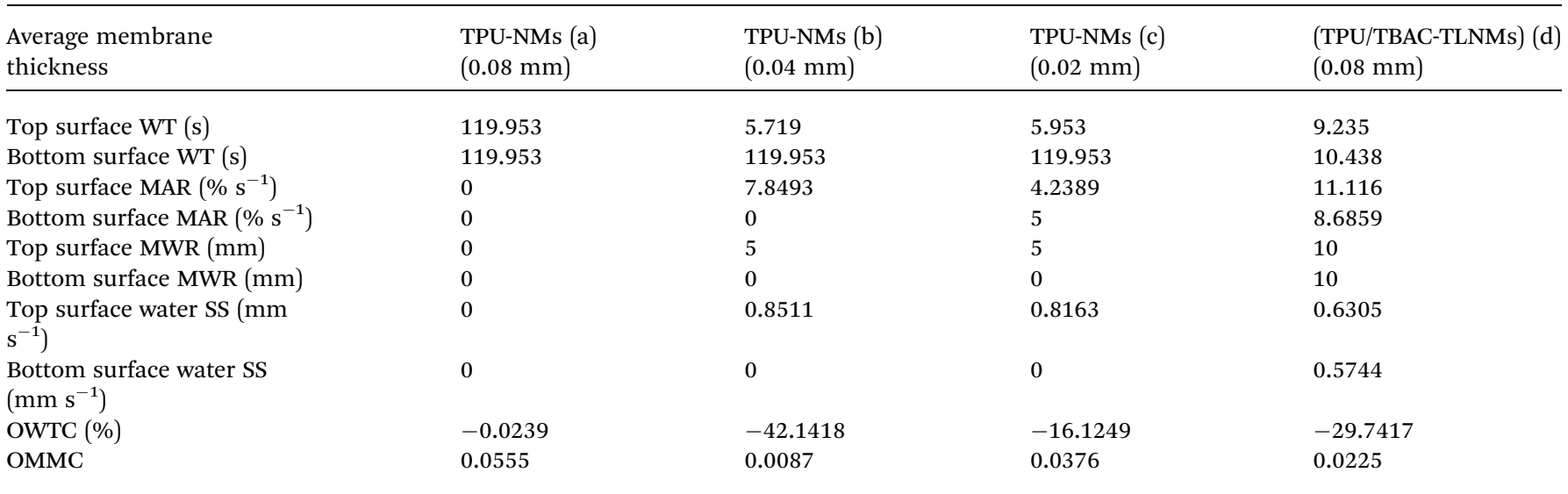
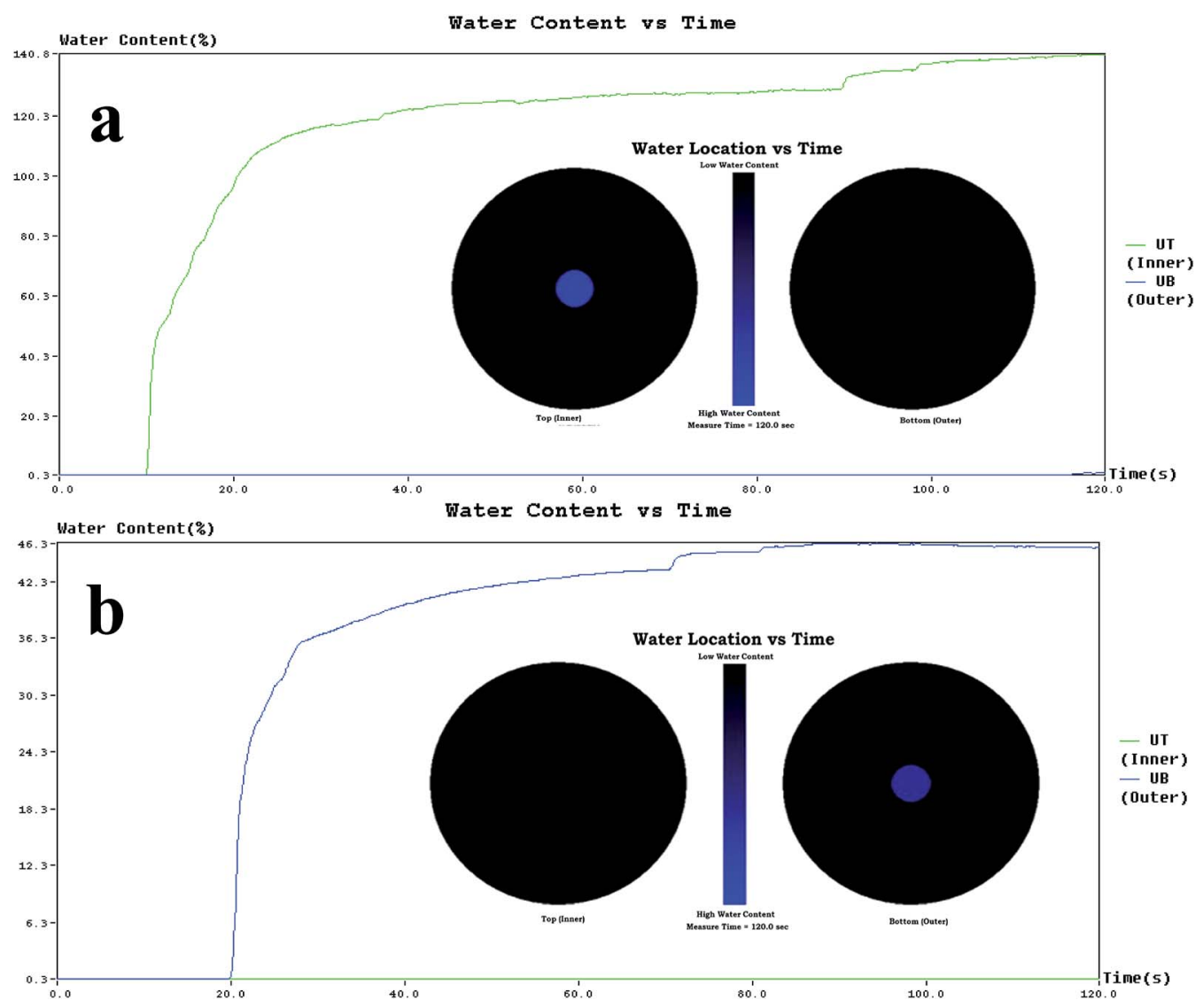

Fig. 7 MMT images of the double-layer membrane: (a) the water was dripped to the TPU/TBAC-TLNMs side and (b) the water was dripped to the TPU-NMs side.

have fine fiber diameters and rough membrane surfaces. Moreover, the friction coefficient of the TPU-NMs side in the double-layer membrane $(0.49)$ is a little lower than that of the TPU-NMs single layer (0.51), because the TPU-NMs side in the double-layer membrane was directly taken from aluminium foil on a rotating receptor and has a smooth membrane surface.

\section{The washable performance analysis}

The washing resistance experiment was used to test the modeholding nature of the membranes after washing. The length and width of the membranes before and after washing is shown in Table 3. The difference in the width measurements for the TPU-NMs was less than $2 \%$ before and after washing, so they had good shape maintenance. This was because the water could not pass into the hydrophobic polyurethane TPU-NMs and 
Table 2 MMT test data for the double-layer membrane

\begin{tabular}{|c|c|c|}
\hline $\begin{array}{l}\text { Average membrane } \\
\text { thickness }\end{array}$ & $\begin{array}{l}0.12 \mathrm{~mm} \text { the water was } \\
\text { dribbled to the } \\
\text { TPU/TBAC-TLNMs side }\end{array}$ & $\begin{array}{l}0.12 \mathrm{~mm} \text { the water } \\
\text { was dribbled to the } \\
\text { TPU-NMs side }\end{array}$ \\
\hline Top surface WT (s) & 9.562 & 119.953 \\
\hline Lower surface WT (s) & 119.953 & 19.797 \\
\hline $\begin{array}{l}\text { Top surface } \\
\text { MAR }\left(\% \mathrm{~s}^{-1}\right)\end{array}$ & 8.2155 & 0 \\
\hline $\begin{array}{l}\text { Lower surface } \\
\operatorname{MAR}\left(\% \mathrm{~s}^{-1}\right)\end{array}$ & 0 & 8.3549 \\
\hline $\begin{array}{l}\text { Top surface } \\
\text { MWR (mm) }\end{array}$ & 5 & 0 \\
\hline $\begin{array}{l}\text { Lower surface } \\
\text { MWR (mm) }\end{array}$ & 0 & 5 \\
\hline $\begin{array}{l}\text { Top surface } \\
\text { water SS }\left(\mathrm{mm} \mathrm{s}^{-1}\right)\end{array}$ & 0.5145 & 0 \\
\hline $\begin{array}{l}\text { Lower surface } \\
\text { water SS }\left(\mathrm{mm} \mathrm{s}^{-1}\right)\end{array}$ & 0 & 0.2506 \\
\hline OWTC (\%) & -111.5435 & 34.9324 \\
\hline OMMC & 0 & 0.0944 \\
\hline
\end{tabular}

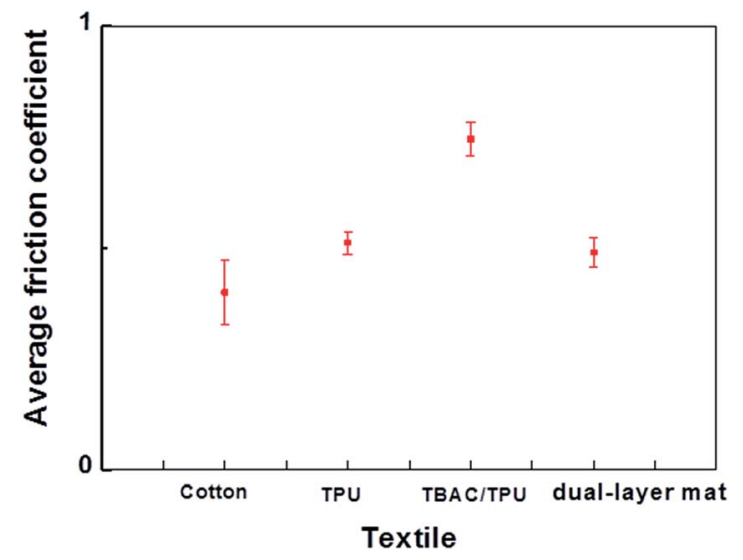

Fig. 8 The friction coefficients of cotton cloth, TPU-NMS, TPU/TBACTLNMs and the double-layer membranes.

cause breakage and deformation of the nanofibers. The hydrophilic TPU/TBAC-TLNMs absorbed some of the water because of the capillary effect, and the contained salt was dissolved which made the size decrease. However, the double-layer membrane had good shape maintenance because of the support of the TPU-NMs layer, and the difference was also less than $2 \%$ before and after washing. The thickness of a membrane before and after washing was tested by taking an average over 20 points as shown in Fig. 9. The results follow the same principle as observed for the length and width measurements, which verified that the double-layer membrane could reduce the loss in thickness caused by washing.

The water vapour transmission (WVT) rate, moisture permeability, hydrostatic pressure, air permeability and filtration efficiency of the double-layer membrane before and after washing are presented in Table 4 . Because the thickness of the double-layer membrane was decreased after washing, these performances all changed: the WVT rate increased from $2170 \mathrm{~g}$ $\mathrm{m}^{-2} \mathrm{~d}^{-1}$ to $2999 \mathrm{~g} \mathrm{~m}^{-2} \mathrm{~d}^{-1}$; the hydrostatic pressure decreased from $10000 \mathrm{~mm} \mathrm{Aq}$ to $7600 \mathrm{~mm} \mathrm{Aq}$; air permeability increased from $1.16 \mathrm{~L} \mathrm{~m}^{-2} \mathrm{~s}^{-1}$ to $1.75 \mathrm{~L} \mathrm{~m}^{-2} \mathrm{~h}^{-1}$; and filtration efficiency decreased from $99.95 \%$ to $99.91 \%$. A normal adult loses $700-$ $1200 \mathrm{~g}$ each day in sweat in the leisure state (insensible perspiration), and 1200-2000 $\mathrm{g}$ each day in the motion state (sensible perspiration). Therefore, the wearing comfort of clothing should be guaranteed when the moisture transmission reaches $2000 \mathrm{~g} \mathrm{~m}^{-2} \mathrm{~d}^{-1}$. According to these standards, all the indexes of the double-layer membrane before and after washing could meet the needs of comfort and protection.

\section{The comparison with the commercial membrane}

The moisture permeability, hydrostatic pressure and air permeability of both the double-layer membrane and a commercial PU waterproof breathable membrane are shown in Fig. 10. The WVT rate, hydrostatic pressure and air permeability of the double-layer membrane are $2170 \mathrm{~g} \mathrm{~m}^{-2} \mathrm{~d}^{-1}, 10000$ $\mathrm{mm} \mathrm{Aq}$ and $1.16 \mathrm{~L} \mathrm{~m}^{-2} \mathrm{~s}^{-1}$, respectively, which are significantly better than the measurements for the commercial PU waterproof breathable membrane $\left(1880 \mathrm{~g} \mathrm{~m}^{-2} \mathrm{~d}^{-1}, 9300 \mathrm{~mm} \mathrm{Aq}\right.$ and $0.302 \mathrm{~L} \mathrm{~m}^{-2} \mathrm{~s}^{-1}$ ). The advantages could be explained by the fact that the double-layer membrane prepared by electrospinning has a porous structure, which brings better performance than the commercial film in terms of moisture and air permeability. Moreover, the hydrophilic surface (TPU/TBAC-TLNMs) of the double-layer membrane has good water-retaining properties, which prevents the hydrophobic surface (TPU-NMs) from coming into direct contact with water, and thus the hydrostatic pressure of the double-layer membrane is high. Therefore, the double-layer membrane offers commercial value due to its performance.

Table 3 The lengths and widths of different membranes before and after washing

\begin{tabular}{|c|c|c|c|c|c|}
\hline Name & Washing & Warp-direction & Transverse & Warp-direction & Transverse \\
\hline & After & 10.1 & 9.8 & 10.0 & 10.0 \\
\hline \multirow[t]{2}{*}{ TBAC/TPU membranes } & Before & 10.0 & 10.0 & 10.0 & 10.0 \\
\hline & After & 10.3 & 9.5 & 9.9 & 9.9 \\
\hline
\end{tabular}



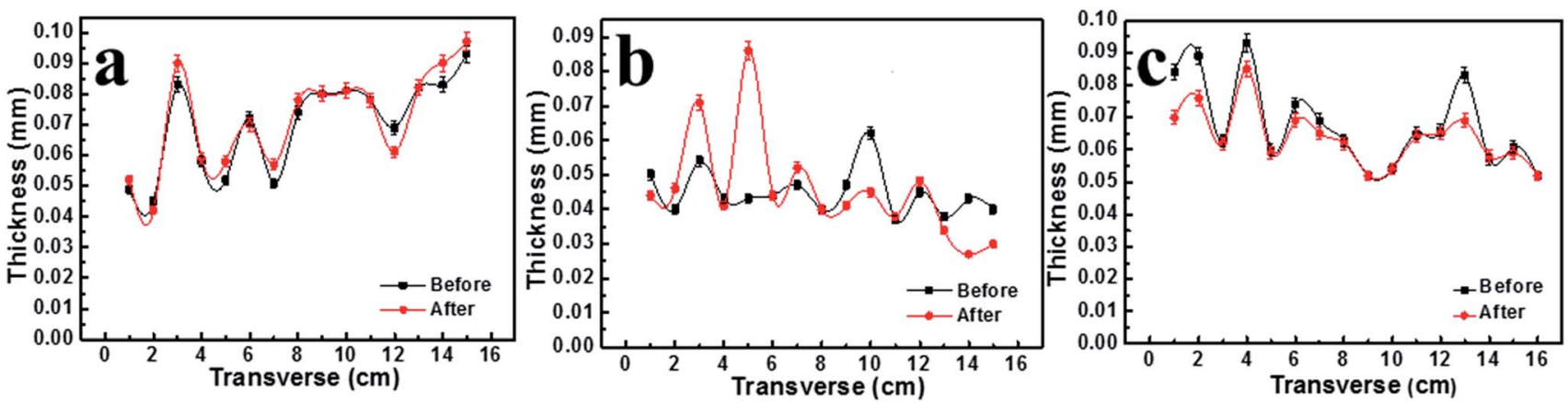

Fig. 9 The thicknesses of the membranes before and after washing: (a) TPU-NMs, (b) TPU/TBAC-TLNMs and (c) the double-layer membrane.

Table 4 WVT rate, moisture permeability, hydrostatic pressure, air permeability and filtration efficiency of the double-layer membrane before and after washing

\begin{tabular}{llllll}
\hline Name & Washing & $\begin{array}{l}\text { WVT rate } \\
\left(\mathrm{g} \mathrm{m}^{-2} \mathrm{~d}^{-1}\right)\end{array}$ & $\begin{array}{l}\text { Hydrostatic pressure } \\
(\mathrm{mm} \mathrm{Aq})\end{array}$ & $\begin{array}{l}\text { Air permeability } \\
\left(\mathrm{L} \mathrm{m}^{-2} \mathrm{~s}^{-1}\right)\end{array}$ & $\begin{array}{l}\text { Filtration efficiency } \\
(\%)\end{array}$ \\
\hline Double-layer membranes & Before & 2170 & 10000 & 1.16 & 99.95 \\
& After & 2999 & 7600 & 1.75 & 99.91
\end{tabular}
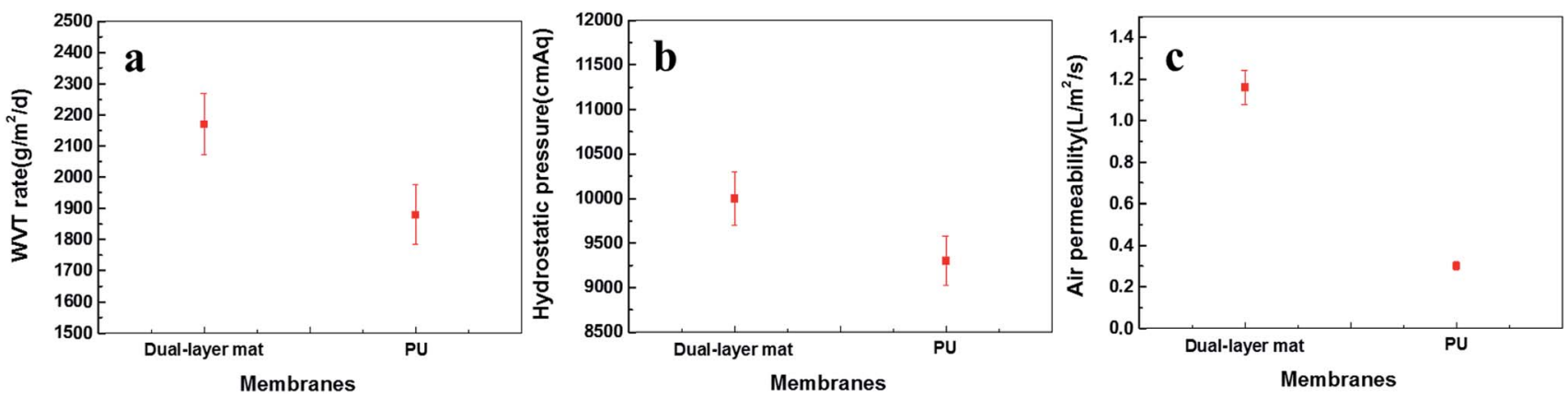

Fig. 10 Comparison of the double-layer membrane and a commercial PU waterproof breathable membrane with respect to (a) moisture permeability, (b) hydrostatic pressure and (c) air permeability.

\section{Conclusions}

A double-layer membrane (TPU-NMs inside and TPU/TBACTLNMs outside) was successfully prepared by direct combination using electrospinning. The different sides of the double-layer membrane presented various micro-structures and performances. The inside (TPU-NMs) was hydrophobic (contact angle = $116.5^{\circ}$ ), and the outside (TPU/TBAC-TLNMs) was hydrophilic (contact angle $=71.5^{\circ}$ ). The double-layer membrane had a pore size distribution of $0.4-1.8 \mu \mathrm{m}$ (mainly in the 0.4 to $0.6 \mu \mathrm{m}$ range), a breaking strength of $2.9 \mathrm{MPa}$, a friction coefficient of 0.49 (similar to that of cotton cloth) and therefore good comfort for clothing. The total unidirectional overall wet transport capacity of the double-layer membrane was $34.9324 \%$, which fully demonstrated its unidirectional moisture transporting properties. Moreover, the double-layer electrospinning membrane offered obvious advantages compared with a commercial PU waterproof breathable membrane.

\section{Acknowledgements}

This work was supported by the National Natural Science Foundation of China $(51673148,51678411)$, the National Key Technology Support Program (2015BAE01B03), the Science and Technology Plans of Tianjin (16PTSYJC00110, 16JCTPJC45600, 15PTSYJC00230) and the National Key Research and Development Project (2016YFB0303304).

\section{Notes and references}

1 Y. Li, Z. Zhu, J. Yu and B. Ding, ACS Appl. Mater. Interfaces, 2015, 7, 13538.

2 F. F. Yang, Y. Li, X. Yu, G. N. Wu, X. Yin, J. Y. Yu and B. Ding, RSC Adv., 2016, 6, 87820.

3 X. Li, X. Yu, C. Cheng, L. Deng, M. Wang and X. Wang, ACS Appl. Mater. Interfaces, 2015, 7, 21919. 
4 J. L. Sheng, M. Zhang, W. J. Luo, J. Y. Yu and B. Ding, RSC Adv., 2016, 6, 29629.

5 A. Gugliuzza and E. Drioli, J. Membr. Sci., 2013, 446, 350.

6 J. F. Ge, Y. Si, F. Fu, J. L. Wang, J. M. Yang, L. X. Cui, B. Ding, J. Y. Yu and G. Sun, RSC Adv., 2013, 3, 2248.

7 T. Subbiah, G. S. Bhat, R. W. Tock, S. Parameswaran and S. S. Ramkumar, J. Appl. Polym. Sci., 2009, 96, 557.

8 X. F. Wang, B. Ding, J. Y. Yu and M. R. Wang, Nano Today, 2011, 6, 510.

9 X. Pan, Z. Wang, Z. Cao, S. Zhang, Y. He, Y. Zhang, K. Chen, Y. Hu and H. Gu, Smart Mater. Struct., 2016, 25, 105010.

10 F. Zhang, Z. Zhang, Y. Liu and J. Leng, Smart Mater. Struct., 2014, 23, 65020.

11 Y. Li, F. F. Yang, J. Y. Yu and B. Ding, Adv. Mater. Interfaces, 2016, 3, 1600516.

12 J. L. Lowery, N. Datta and G. C. Rutledge, Biomaterials, 2010, 31, 491.

13 W. E. Teo and S. Ramakrishna, Nanotechnology, 2006, 17, R89.

14 A. L. Yarin and E. Zussman, Polymer, 2004, 45, 2977.

15 N. E. Zander, Polymer, 2013, 5, 19.

16 Z. M. Huang, Y. Z. Zhang, M. Kotaki and S. Ramakrishna, Compos. Sci. Technol., 2003, 63, 2223.

17 B. Ding, W. Moran, X. Wang, J. Yu and G. Sun, Mater. Today, 2010, 13, 16.

18 K. Sureeporn, W. Liu and D. H. Reneker, J. Polym. Sci., Part B: Polym. Phys., 2001, 39, 2598.

19 M. Xia, Q. Liu, Z. Zhou, Y. Tao, M. F. Li, K. Liu, Z. Wu and D. Wang, J. Power Sources, 2014, 266, 29.

20 N. T. Xuyen, T. H. Kim, H.-Z. Geng, H. Lee, K. K. Kim and Y. H. Lee, J. Mater. Chem., 2009, 19, 7822.
21 M. Shang, W. Wang, L. Zhang, S. Sun, L. Wang and L. Zhou, J. Phys. Chem. C, 2009, 113, 14727.

22 J. Lin, B. Ding, J. Yang, J. Yu and G. Sun, Nanoscale, 2012, 4, 176.

23 L. Ge, X. Wang, Z. Tu, C. Pan, C. Wang and Z. Gu, Jpn. J. Appl. Phys., Part 1, 2007, 46, 6790.

24 Y. K. Kang, C. H. Park, J. Kim and T. J. Kang, Fibers Polym., 2007, 8, 564.

25 L. Sumin, D. Kimura, A. Yokoyama, K. H. Lee, J. C. Park and I. S. Kim, Text. Res. J., 2009, 79, 1085.

26 L. Sumin, D. Kimura, K. H. Lee, J. C. Park and I. S. Kim, Text. Res. J., 2010, 80, 99.

27 B. Yoon and S. Lee, Fibers Polym., 2011, 12, 57.

28 A. Nishimoto, Y. Omote, K. Takahashi and A. Nakagawa, Sen'i Gakkaishi, 2003, 59, 344.

29 Y. Dong, J. Kong, S. L. Phua, C. Zhao, N. L. Thomas and X. Lu, ACS Appl. Mater. Interfaces, 2014, 6, 14087.

30 Z. Li, Y. Xu, L. Fan, W. Kang and B. Cheng, Mater. Des., 2016, $92,95$.

31 L. W. Zhang, Y. Li, J. Y. Yu and B. Ding, RSC Adv., 2015, 5, 79807.

32 X. H. Qin, Y. Q. Wan, J. H. He, J. Zhang, J. Y. Yu and S. Y. Wang, Polymer, 2004, 45, 6409.

33 X. H. Qin, L. Jia and W. Y. Lu, Text. Res. J., 2011, 81, 388.

34 Z. J. Li, W. M. Kang, N. Wei, J. A. Qiu, C. Sun and B. W. Cheng, RSC Adv., 2017, 7, 8220.

35 Y. Dong, J. Kong, C. Mu, C. Zhao, N. L. Thomas and X. Lu, Mater. Des., 2015, 88, 82.

36 H. Li, W. Shi, Y. Zhang, Q. Du, X. Qin and Y. Su, Sep. Purif. Technol., 2016, 166, 240. 